\title{
Retrouver le paysage
}

\author{
Intérêt, sources et méthodes \\ Thibault Bechini, Grégoire Binois, Noëmie Lucas, \\ Clara Stevanato
}

Retrouver le paysage, c'est faire une double histoire : celle d'un objet et celle d'un concept ${ }^{1}$. Par objet, il faut entendre la perception située d'un environnement changeant ; par concept, un outil que le chercheur peut mobiliser pour interroger ce qui n'est plus. La perte du paysage est souvent liée à plusieurs phénomènes : la destruction - ou la modification - de ses marqueurs physiques, la mutation des perceptions que l'on en a, ou encore le changement de positionnement des historiens qui, ces dernières années, se sont tournés vers d'autres objets ${ }^{2}$. Ce dossier propose donc de réinvestir le concept de paysage, de le "retrouver ${ }^{3}$ " et de rappeler la pertinence de son étude à un moment où les rapports homme/milieu sont principalement saisis à travers le prisme de l'histoire environnementale. Le paysage est pourtant un objet qui échappe à l'historien. Jamais il ne sera en mesure de faire revivre la complexité d'une configuration environnementale, avec ses dynamiques et les multiples regards qui l'envisagent. Il ne peut qu'en proposer une modélisation partielle et partiale, à l'instar des acteurs ayant produit les sources qu'il utilise. Cependant, en tentant de retrouver - partiellement - le paysage, les articles se donnent les moyens de saisir les rapports homme/milieu et les processus qui les façonnent. Plutôt que de considérer le paysage comme un tableau immobile, elles insistent sur le caractère mouvant de ce dernier et sur la perception de ses transformations. Penser en termes de paysage permet donc de travailler sur l'appréhension des dynamiques environnementales.

1. Nous tenons à remercier tous les professeurs qui nous ont relus, conseillés et accompagnés pour cette journée doctorale : Christine Lebeau, François Chausson et Grégory Quenet, nos directeurs de thèse respectifs ainsi que Christine Ducourtieux et Noemi Rubello.

2. Voir infra.

3. Voir A. Antoine, Le Paysage de l'historien. Archéologie des bocages de l'Ouest de la France à l'époque moderne, Rennes, 2002, et plus particulièrement les sections "L'effacement du paysage » (p. 21-26) et "On a retrouvé le paysage » (p. 38-40). 


\section{Le paysage et l'École doctorale : une histoire de vingt ans}

Retrouver le paysage est donc, avant tout, une injonction historiographique. Il s'agit de remettre en avant un concept concurrencé ces dernières années par d'autres clefs d'analyse (environnement, nature, territoire, etc.). Toutes ont en commun de porter sur les relations entre l'homme et son milieu. Elles en proposent cependant des approches distinctes. Afin de saisir ces déplacements méthodologiques et de replacer ainsi le dossier dans son contexte de production, une analyse des récentes publications d'Hypothèses semble judicieuse. Ces cahiers, dans lesquels paraît le présent dossier, sont en effet le reflet d'une historiographie située, autrement dit occidentale et, plus encore, parisienne ${ }^{4}$. Ils permettent d'envisager l'environnement culturel dans lequel nous évoluons.

En 1998, Samuel Leturcq et d'autres doctorants ${ }^{5}$ constituaient un dossier consacré au paysage. Notre proposition de "Retrouver le paysage " s'inscrit en dialogue avec ce premier numéro de la revue. Depuis ce séminaire, dont le principal objectif était de « faire sauter les cloisonnements universitaires et disciplinaires entre historiens, archéologues et géographes » afin de « donner une interprétation particulière d'un paysage en fonction de sa sensibilité disciplinaire ou des sources ${ }^{6}$ " utilisées, plusieurs autres dossiers, au cours de ces deux décennies, ont placé les thématiques paysagères au cœur de leur réflexion, sans pour autant en reprendre le terme : en 2001, le séminaire «L'espace et l'histoire »; en 2005 «L'appropriation du territoire par les communautés "; en 2010, "Posséder la terre "; en 2012, " Habiter un espace " et, surtout, en 2014, le séminaire qui s'intitulait « Nature et société ».

Le thème du paysage est donc sous-jacent à de nombreuses publications, mais ces dernières n'en font pas toujours le point central de leur argumentation. Analyser la manière dont ces questions ont été abordées lors de ces différentes rencontres permet d'envisager un certain nombre d'évolutions historiographiques.

4. La catégorie de paysage est vécue et utilisée différemment ailleurs, comme en Asie, ce qu'a montré notamment Augustin Berque pour la Chine antique : A. Berque, La pensée paysagère, Paris, 2008.

5. Il s'agit d'Anne Mailloux, de François Duceppe-Lamarre et de Laurent Lespez. La conclusion était réalisée par Patrice Beck.

6. S. LeturcQ, «Le paysage : introduction », Hypothèses, 1 (1999), p. 71-74. 
En 1998, dans l'introduction de Samuel Leturcq ${ }^{7}$, la définition retenue pour le paysage consistait en « la vision qu'un individu peut avoir d'un environnement en particulier ", autrement dit, un objet vu, une réalité matérielle perçue : il s'agissait, avant tout, d'une "représentation de l'espace ». Pour autant, les interventions proposées par le géographe ${ }^{8}$ et l'archéologue ${ }^{9}$ de l'équipe, Laurent Lespez et François Duceppe-Lamarre, définissaient déjà le paysage comme une réalité complexe, qui était le produit de l'interaction entre des phénomènes naturels - ou faits de nature - et des actions anthropiques - ou faits de société.

Deux lignes de force se dégageaient de ce séminaire. Il s'agissait, d'une part, de favoriser la rencontre interdisciplinaire - ce qui ne sera pas notre perspective - et d'autre part, d'étudier les paysages dans leur dimension dynamique. Les interventions des doctorants reflétaient, ainsi que le rappelait Patrice Beck ${ }^{10}$, les orientations énoncées par Georges Bertrand dans L'Histoire de la France rurale ${ }^{11}$, qui privilégiaient l'étude des interactions entre l'homme et son milieu dans le contexte de la montée en puissance de l'écologie et de la problématique environnementale. Cette dynamique de recherche s'est alors traduite, dans le champ éditorial, par le lancement par la maison d'édition Champ Vallon d'une collection centrée sur le paysage ${ }^{12}$, suivie d'une deuxième collection intitulée "L'environnement a une histoire ", dirigée par Grégory Quenet.

Cette préoccupation ruraliste se retrouvait dans le séminaire " L'espace et l'histoire", en 2001. Voulant ériger les relations homme-milieu en domaine d'étude, les auteurs replaçaient le paysage dans le sillage de l'histoire rurale, qui s'intéressait notamment aux structures agraires que le paysage enregistrait ${ }^{13}$. Élargissant le corpus documentaire par la consultation

\section{Ibid.}

8. L. Lespez, "De la morphogenèse aux paysages : un exemple de recherche de géographie physique en Grèce du Nord ", Hypothèses, 1 (1999), p. 75-83.

9. F. Duceppe-Lamarre, "L'archéologie du paysage à la conquête des milieux forestiers, ou l'objet paysage vu par l'archéologue de l'environnement ", Hypothèses, 1 (1999), p. 85-94.

10. P. Beck, «Le paysage : conclusion ", Hypothèses, 1 (1999), p. 115-118.

11. G. Bertrand, "Pour une histoire écologique de la France rurale ", dans Histoire de la France rurale, G. Duby et A. WAllon dir., Paris, 1975-1976, t. 1, p. 37-113.

12. Il s'agit de la collection " Pays Paysages".

13. M. SAUdAn, "Géographie historique. Histoire d'une discipline controversée ou repères historiographiques", Hypothèses, 5 (2002), p. 13-25 (p. 22). 
des sources cadastrales, le dossier " Posséder la terre » de 2010 considérait l'espace comme le résultat de processus sociaux ${ }^{14}$. D'une manière générale, il semble que l'une des problématiques centrales de presque tous ces séminaires - « L'appropriation du territoire ", " Posséder la terre ", " Habiter un espace » et, pour certaines de ses communications, « Nature et société » - fût l'appropriation du paysage par l'homme.

Le séminaire "Nature et société " se distingue néanmoins des précédents en ce qu'il propose, en 2014, d'examiner la manière dont les historiens ont pensé les rapports entre société et nature. C'est dans le cadre de cet essai d' 'épistémologie historique ${ }^{15}$ " que le paysage est évoqué, afin de rendre compte des apports et des limites théoriques de l'analyse constructiviste. Cette analyse part du principe que ce qu'on appelle "nature " est une construction sociale, donc une réalité qui n'est connaissable qu’à travers des catégories préalables. Dans l'introduction, le concept de " paysage » est envisagé comme une étape réflexive dans l'historicisation du rapport entre nature et société. Il renvoie ainsi à une compréhension anthropocentrique des rapports nature-société, dans laquelle le chercheur ne viserait pas à faire l'histoire des mutations paysagères - comme précédemment -, mais à analyser « tout ce qui influe sur la façon de charger l'espace de signification, de symboles et de désir ${ }^{16}$ ».

\section{Dans le prolongement des réflexions d'Angelo Torre: le paysage comme processus d'activation de ressources}

La définition du paysage pour laquelle nous avons opté tient compte des apports des travaux culturalistes, en soulignant le processus de construction des paysages à travers les âges. Elle vise également à renouer avec une approche à l'échelle topographique ${ }^{17}$. Dans cette optique, notre intérêt s'est porté sur les dynamiques anthropiques et naturelles à l'œuvre dans la production du paysage. Ce dernier est alors entendu comme une portion de réel irréductible aux seules perceptions panoramiques que nous offrent la peinture, la photographie ou les descriptions littéraires. La vue d'ensemble

14. M. Cerovic et al., "Posséder la terre. Approches et enjeux historiographiques ", Hypothèses, 14 (2011), p. 163-179 (p. 173).

15. L. Teixeira, "Nature et société. Éléments d'une épistémologie historique ", Hypothèses, 18 (2015), p. 17-28.

16. A. Corbin, L'homme dans le paysage, Paris, 2001, p. 57.

17. The Iconography of Landscape: Essays on the Symbolic Representation, Design and Use of Past Environments, S. Daniels et D. E. Cosgrove dir., Cambridge, 1988. 
ne saurait suffire. Il faut souvent procéder à l'agencement de fragments de nature diverse, pour tenter de retrouver le paysage dans toute sa complexité.

Portion de réel dont les sources ne donnent souvent qu'une vision partielle, le paysage peut être défini comme le « résultat des pratiques d'activation des ressources", pour reprendre les termes de l'historien Angelo Torre qui, dans un article paru en 2008 dans les Annales, invitait les historiens à ne pas s'en tenir à une utilisation exclusivement visuelle et symbolique de la catégorie de paysage ${ }^{18}$. Dans cet article, deux approches du paysage sont distinguées : l'une, symbolique, qui repose sur une appréhension relativiste et visuelle du paysage ; l'autre, écologique, fondée sur la notion de ressource, qui envisage le paysage dans ses dynamiques historiques précises. En recourant à la notion de « ressource " dans sa définition du paysage, Angelo Torre suggère que les " éléments visuels du paysage " ne sont que "les éléments actifs d'une sélection de la part des groupes humains qui les utilisent ${ }^{19}$ ". Il complète son propos en rappelant que « les pratiques locales activent les ressources de façon sélective ", la définition du paysage résidant dans « l'articulation de ces ressources activées ". Il incombe donc de savoir tenir compte dans nos travaux de ces pratiques et de ces phénomènes, parfois successifs, parfois concomitants, souvent concurrents, qui contribuent à la formation des paysages par l'activation sélective des ressources locales.

En prêtant une attention particulière à la processualité sous-jacente de tout paysage, nous avons souhaité aborder le paysage comme un palimpseste toujours en devenir, dont les formes anciennes ne disparaissent jamais tout à fait et dont les dynamiques naturelles et anthropiques à un temps donné sont si nombreuses que l'on a parfois l'impression d'être en présence de paysages mouvants.

Aussi, en voulant retrouver le paysage, nous avons également souhaité retrouver la richesse heuristique d'une notion qui permet de saisir ensemble les pratiques, les phénomènes et les représentations qui se déploient au sein d'un espace donné. Dans nos articles, nous avons cherché à saisir les logiques de sélection et d'enrichissement de l'information qui fondent toute perception d'un paysage. Notre démarche ne se réduit donc pas à retrouver la matérialité d'une portion de réel, mais consiste, bien davantage, à en faire une étape de travail, afin de mieux poursuivre nos réflexions sur les sociétés humaines des siècles passés.

18. A. Torre, "Un “tournant spatial” en histoire ? Paysages, regards, ressources », Annales. Histoire, sciences sociales, 63/5 (2008), p. 1127-1144.

19. Ibid., p. 1139. 


\section{Un parti pris méthodologique}

Le parti pris des différentes contributions est donc de proposer une approche résolument méthodologique des études paysagères. Il s'agit de dépasser l'approche culturaliste (fondée sur ce que les sources sont, à savoir une lecture de l'espace, produite par une société donnée en sélectionnant les éléments jugés pertinents), pour envisager la matérialité du paysage (dont les sources ne nous donnent toujours qu'une vision tronquée). C'est en effet la volonté de confronter ces deux composantes du paysage, l'une physique, l'autre culturelle, qui nous rassemble. Face à un paysage disparu, uniquement décrit par des sources qui n'en documentent que certains aspects, l'historien, s'il ne veut pas être dupe des effets de réels induits, doit s'atteler à un important travail de déconstruction des représentations paysagères. $\mathrm{Ce}$ n'est que par ce travail préalable, mais dont les résultats sont importants en eux-mêmes, qu'il peut approcher le paysage passé. En croisant les sources et en prenant conscience des logiques sous-jacentes de sélection et de présentation de l'information, les différentes contributions se donnent ainsi les moyens d'appréhender la matérialité d'un terrain et d'analyser les filtres perceptifs qui s'y ajoutent.

Mais les quatre articles prêtent également attention aux processus qui participent à l'établissement du paysage. Cette processualité, c'est à dire la dynamique intrinsèque aux paysages, est liée aux modifications matérielles de l'espace (l'urbanisation, l'assèchement des marais et, plus généralement, l'ensemble des dynamiques hydrographiques sont par exemple régulièrement invoqués), mais s'appuie également sur les évolutions perceptives et culturelles. L'édification d'un temple ou la reconquête d'une terre cultivable change le regard porté sur l'espace. Il en modifie le sens et la valeur. Le paysage devient donc cette réalité hybride, ce concept permettant de saisir en même temps et de manière dynamique le donné matériel et sa représentation, l'espace et l'acteur social qui le perçoit. Il représente donc un outil pertinent pour étudier l'inscription des hommes dans l'espace.

\section{Des sources aux paysages}

Travailler à retrouver des paysages nécessite de mener une réflexion critique sur les sources qui les saisissent. Le positionnement historiographique vis-à-vis de la catégorie de paysage est lié à un positionnement méthodologique vis-à-vis de la documentation. 
Les sources du paysage, quelles qu'elles soient, reposent en effet sur une lecture spatiale préalable. Aucune ne donne directement accès à la matérialité d'une portion de réel. Lorsqu'un document l'évoque, c'est toujours au travers d'un prisme perceptif : celui de son producteur. Les descriptions ne fournissent ainsi qu'une image partielle et biaisée de la réalité qu'elles se proposent de saisir. Il faut donc les critiquer d'abord pour les croiser ensuite.

Parmi les documents utiles à la reconstruction d'un paysage, certains en donnent d'emblée une représentation intégrale et unifiée. Il s'agit par exemple des productions littéraires, iconographiques ou cartographiques. Proposant une "vue ", elles présentent les éléments en système. Mais ces descriptions ne sont jamais qu'une activation d'éléments sélectionnés. Elles répondent à des objectifs et à des sensibilités socialement situés, comme le montrent Thibault Bechini avec l'aridité provençale et Noëmie Lucas avec les marais bas-iraqiens. D'autres sources ne documentent que des objets circonscrits, relativement isolés, sans prétendre fournir une vue d'ensemble. Ces documents, souvent plus techniques, sont principalement liés à des pratiques concrètes de l'espace comme le cadastre, les rapports de bornage, voire les bornes elles-mêmes. Si la production de ces sources est elle aussi biaisée, ces documents présentent néanmoins l'avantage de ne pas avoir été conçus pour offrir une vision paysagère. Les informations pertinentes pour une étude du paysage y sont donc présentées sous un jour différent. Le croisement de ces deux types de documents, de ces deux types de regards portés sur l'espace, est souvent très efficace pour approcher les paysages anciens.

Mais ces informations gagnent également à être intégrées dans une perspective diachronique permettant de souligner les processus à l'œuvre dans l'élaboration des paysages. Aux mutations des regards s'ajoute la mutation des objets. Ainsi, les trois dimensions du paysage - culturelle, matérielle, processuelle - vont systématiquement être abordées, mais dans des proportions variables selon l'abondance et la nature de la documentation disponible.

L'iconographie, notamment la photographie et la peinture, est par exemple largement exploitée par Thibault Bechini dans son article. Ces documents contribuent à alimenter un discours folklorique sur le paysage provençal, parfois au service de discours politiques. Pourtant, en s'appuyant sur des sources complémentaires, comme les relevés topographiques et les rapports de bornage, Thibault Bechini parvient à reconstituer les processus d'urbanisation et d'industrialisation qui affectent progressivement l'espace, tout en étant largement occultés par les représentations iconographiques. Son article analyse ainsi les mutations paysagères à l'œuvre dans la banlieue 
marseillaise, pointant le fossé qui se creuse progressivement entre la matérialité d'un espace en cours d'urbanisation et le développement d'une représentation essentialisée et conservatrice de la campagne provençale, au tournant du $\mathrm{Xx}^{\mathrm{e}}$ siècle.

En prenant l'exemple de la Basse-Alsace du XviII ${ }^{\mathrm{e}}$ siècle telle qu'elle peut être saisie par les sources militaires, Grégoire Binois fait du paysage un concept utile pour penser l'inscription de la guerre dans l'espace. Convoquant cartes et mémoires d'ingénieurs, il montre comment les impératifs militaires impliquent une façon spécifique d'appréhender le paysage et d'y intégrer les activités civiles. Interrogeant lui aussi la processualité, il montre que les espaces frontaliers sont, en réalité, caractérisés par l'organisation d'une alternance paysagère.

Noëmie Lucas, en mobilisant des sources littéraires plurielles (encyclopédie et ouvrages de géographie administrative), tente de retrouver les lectures d'un paysage qui, par sa nature mouvante, est difficilement saisissable. En analysant les processus paysagers à l'œuvre dans les marais bas-iraqiens au travers de l'étude de la fabrication des discours, elle cherche à montrer que la nature et les modalités de la description de cet espace sont commandées, au début de l'époque islamique, par les caractéristiques du paysage décrit.

Clara Stevanato propose, enfin, de saisir le paysage à partir des sources épigraphiques. Ces documents, concernant l'aristocratie romaine à l'époque impériale, permettent d'établir les différentes fonctions de l'espace, de repérer, par exemple, des propriétés ou des sépultures et de documenter ainsi leurs dimensions matérielles et culturelles. Ce faisant, l'article met particulièrement bien en lumière, du fait de la difficile conservation des sources, l'aspect profondément parcellaire de toute reconstitution paysagère, procédant par accumulation de données ponctuelles. 